J. Lake Sci. (湖泊科学), 2015, 27(2):335-342

http: //www. jlakes. org. E-mail : jlakes@niglas.ac.cn

(c) 2015 by Journal of Lake Sciences

\title{
遥感影像空间分辨率变化对湖泊水体提取精度的影响"
}

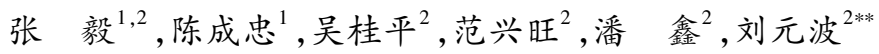 \\ ( 1 : 湖北师范学院城市与环境学院, 黄石 435002$)$ \\ (2: 中国科学院南京地理与湖泊研究所,南京 210008)
}

\begin{abstract}
摘 要: 湖泊面积是表征湖泊水情变化的重要指示因子,如何从不同空间分辨率遥感数据中获取客观准确的水面信息, 是当前遥感应用研究中的难点问题. 本文以鄱阳湖为例,通过选用丰水期和枯水期代表性 Landsat ETM + 遥感影像, 采用 最邻近法 $(\mathrm{NN})$ 和像元聚合法 $(\mathrm{PA})$ 两种重采样方法, 分别获取分辨率逐渐降低的不同分辨率的影像数据, 结合归一化差 异水指数法研究水域面积随遥感影像分辨率降低的变化趋势及其误差变化特征, 同时深人分析不同影响因素对水体提 取精度的差异. 研究结果表明: (1) 空间分辨率是影响鄱阳湖水体提取精度的重要因素之一,随着遥感影像空间分辨率的 降低, 提取水域面积的精度相对 $30 \mathrm{~m}$ 分辨率时呈逐渐降低的趋势, 但整体精度较高, 最低精度在 $67.64 \%$ 以上; (2) NN 重采样方法对遥感影像波段亮度值的均值影响不大, 但 PA 重采样后影像的均值和标准差随分辨率逐渐降低且变化更有 规律; (3) 水体阈值在 PA 重采样后变化较大, NN 重采样后变化较小,因而采用 $30 \mathrm{~m}$ 分辨率时获取的阈值提取 PA 重采 样后鄱阳湖水体误差较大,提取 NN 重采样后的湖泊水体误差较小. 本研究结果对于全球变化影响下湖泊水体信息遥感 精确提取具有重要的参考价值.
\end{abstract}

关键词: 遥感;分辨率;阈值;重采样; NDWI; 鄱阳湖

\section{Effects of spatial scale on water surface delineation with satellite images}

\author{
ZHANG $\mathrm{Yi}^{1,2}$, CHEN Chengzhong ${ }^{1}$, WU Guiping ${ }^{2}$, FAN Xingwang $^{2}$, PAN Xin $^{2} \&$ LIU Yuanbo ${ }^{2}$ \\ (1: College of Urban and Environmental Sciences, Hubei Normal University, Huangshi 435002, P. R. China) \\ (2: Nanjing Institute of Geography and Limnology, Chinese Academy of Sciences, Nanjing 210008, P. R. China)
}

\begin{abstract}
Lake area is an important indicator for lake-related research. Accurate extraction of water surface from remote sensing images with various spatial resolutions still remains unsettled. To investigate the effects of spatial resolution on lake surface monitoring, two Landsat ETM + (Enhanced Thematic Mapper Plus) images were acquired with one at high water level and the other at low water level. These images were resampled to lower resolutions using Nearest Neighboring( NN) and Pixel Aggregation(PA) methods, and extracted for water surface area using NDWI( Normalized Difference Water Index) thresholding method. Variation trend and error distribution of water surface area with spatial resolution were thoroughly investigated. In addition, the influence of different factors on water surface extraction was discussed. The main conclusions are as follows: (1) the accuracy decreases gradually at lower spatial resolution relative to $30 \mathrm{~m}$ resolution. However, the overall accuracy is higher with a minimum of $67.64 \%$; (2) NN has minor impact on DN values, whereas PA reduces mean digital number values and standard values. The reduction is gradual and smoother than NN; and(3) the threshold varies for PA and remains stable for NN. Thus, use of threshold determined on $30 \mathrm{~m}$ image should introduce larger errors for PA-resampled images than NN-resampled images. This study provides invaluable guidance for water surface mapping using remote sensing methods under global changes.
\end{abstract}

Keywords: Remote sensing; spatial resolution; threshold; resampling; NDWI; Lake Poyang

湖泊是陆地表层系统各要素相互作用的节点,在维系区域生态平衡,减轻干旱、洪水灾害等方面发挥着

* 中国科学院南京地理与湖泊研究所“一三五”战略发展规划项目 (NIGLAS2012135001)、国家重点基础研究发展计 划“973”项目 (2012CB417003) 和中国科学院百人计划择优支持项目联合资助. 2014-04-09 收稿; 2014-07-02 收修改稿. 张毅(1988 ), 男, 硕士研究生;E-mail: cqzhangyi318@ 163. com.

** 通信作者;E-mail: ybliu@ niglas. ac.cn. 
不可替代的作用. 近年来, 在全球气候变化和人类活动的影响下, 全球范围内多地区极端干旱、洪水等自然 灾害的频发给社会经济建设和农业生产等都带来了巨大的影响. 由于湖泊面积是表征湖泊受气候变化与人 类活动影响程度的重要指示器, 因此实时准确地提取湖泊水域面积, 对于湖泊周边水资源优化配置、生态环 境保护及其区域可持续发展等意义重大.

卫星遥感具有覆盖范围大、快速、准确的宏观监测能力, 成为近年来湖泊水域面积提取的重要技术方 法 $^{[1-2]}$, 并且得到了越来越多的应用. Birkett ${ }^{[3]}$ 利用卫星雷达测高数据和近红外影像获取了高精度的乍得湖 永久湖泊和洪水淹没区面积; Hui 等 ${ }^{[4]}$ 和 Feng 等 ${ }^{[5]}$ 分别利用 Landsat 数据和 MODIS 数据, 使用归一化差异 水指数 (NDWI) 研究了鄱阳湖的水域面积变化特征. 由于不同空间分辨率的遥感数据所体现的地物特征和 所承载的地物信息量是不同的 ${ }^{[6]}$, 使得借助不同分辨率遥感影像所提取的地表信息不可避免地存在着精度 上的差异. 如何衡量和消除这些差异, 以达到遥感数据最高效的利用, 已经成为当前遥感研究的重要内 容 $^{[7-10]}$, 并且在土地覆盖、地表温度等方面开展了较多研究 ${ }^{[11-13]}$, 然而关于遥感影像空间分辨率变化对湖泊 水体提取精度影响程度、具体变化情况以及不同因素对其产生影响的具体机制方面进行探讨和研究具有很 强的现实意义和价值, 是亟待解决的重要科学问题.

基于此, 本文拟以大型季节性湖泊——鄱阳湖为研究对象, 以具有中高分辨率的 Landsat ETM + 数据为 基础,首先通过选择丰水期和枯水期的典型代表性影像,利用重采样方法获得不同空间分辨率的影像数据, 采用目前被广泛使用的 NDWI 提取对应分辨率的湖泊水体面积, 在此基础上定量分析湖泊水体提取的误差 及其来源, 最终总结出遥感影像空间分辨率变化对湖泊水体提取精度的影响, 以期为湖泊面积的遥感精确 监测提供可靠的方法借鉴和数据保证.

\section{1 数据和方法}

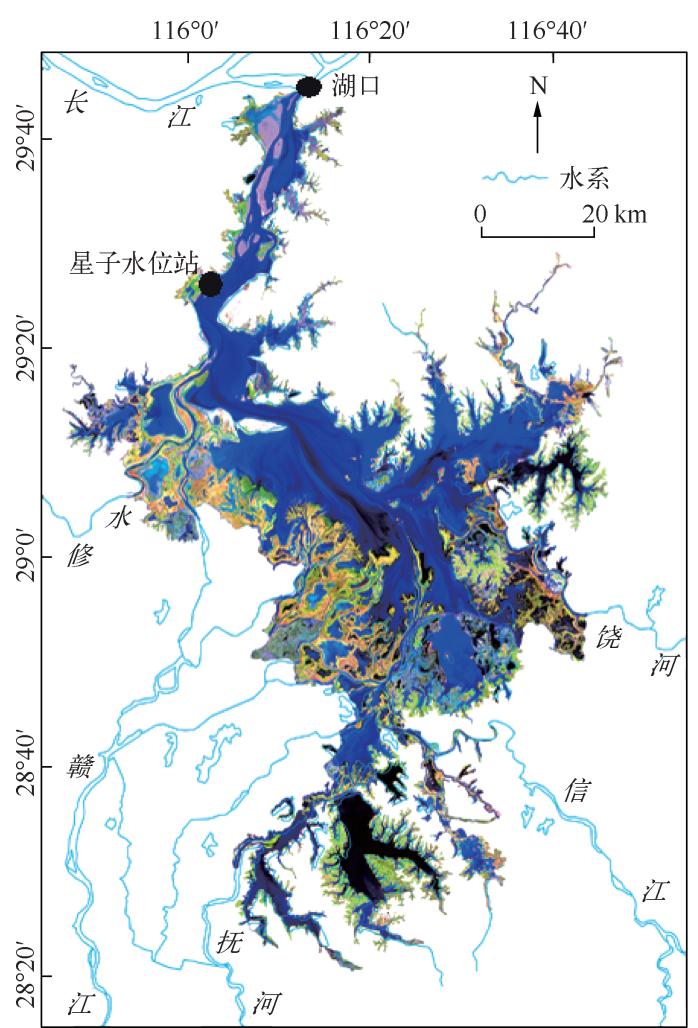

图 1 鄱阳湖区 Landsat ETM+543 波段假彩色合成影像

Fig. 1 Landsat ETM+543 band false color composite images of Lake Poyang

\section{1 研究区概况}

鄱阳 湖 $\left(28^{\circ} 22^{\prime} \sim 29^{\circ} 45^{\prime} \mathrm{N}, 115^{\circ} 47^{\prime} \sim\right.$ $\left.116^{\circ} 45^{\prime} \mathrm{E}\right)$ 是我国最大的淡水湖, 地处江西省北 部. 湖泊主要承纳赣江、抚河、信江、饶河、修水 五河来水, 在涵养水源、调蓄长江洪水、调节气 候及降解污染物等方面发挥着重要作用 ${ }^{[14]}$, 同 时也是补给湖口以下长江干流的主要水源之一 (图 1). 鄱阳湖是一个十分典型的季节性、过水 性和吞吐型浅水湖泊 ${ }^{[15]}$. 湖泊南北最长为 $173 \mathrm{~km}$, 东西最宽为 $70 \mathrm{~km}$, 最窄仅为 $3 \mathrm{~km}$, 平均 宽度 $16.9 \mathrm{~km}^{[16]}$. 湖泊受季风影响, 水域面积变 化明显,在高水位时湖水面积可达 3900 余 $\mathrm{km}^{2}$, 但低水位时仅 100 多 $\mathrm{km}^{2}$, 高水呈湖相, 低水呈 河相, 以致出现“夏秋一水连天, 冬春荒滩无边” 的景象 ${ }^{[17]}$.

\section{2 数据}

本文选择具有中高分辨率的 Landsat 7 $\mathrm{ETM}+($ 空间分辨率为 $30 \mathrm{~m}$ ) 影像作为数据源. 具体操作过程中, 考虑到研究结果的典型性, 有 针对性地选用丰水期 (2000 年 9 月 23 日,星子站 水位: $16.15 \mathrm{~m}$ ) 和枯水期 (2003 年 2 月 20 日,星子 站水位: $12.16 \mathrm{~m}$ ) Landsat ETM + 影像各一景, 分 别抽取出绿光波段(B2) 和近红外波段( B4), 以 进行比值运算处理, 两波段的光谱分辨率分别 
为 $0.52 \sim 0.60 \mu \mathrm{m}$ 和 $0.77 \sim 0.90 \mu \mathrm{m}$. 此外, 为了减小其它误差所带来的影响, 研究过程中对遥感影像数据 不做前期预处理.

\section{3 方法}

本研究的技术路线为: 首先, 在分别抽取丰水期和枯水期 Landsat ETM + 影像 B2、B4 波段的基础上, 选 用两种重采样方法对各自波段进行重采样, 将遥感影像降低到指定分辨率. 然后借助 NDWI, 运用 $30 \mathrm{~m}$ 分辨 率时选取的阈值提取不同分辨率遥感影像的水体. 在此基础上, 分别从相对误差和绝对误差两个方面分析 提取水体的误差随分辨率降低的变化趋势. 接着通过对提取的水体面积随分辨率变化的线性拟合分析, 探 讨分辨率对提取精度的影响, 并从各波段降低分辨率后波段亮度值 (DN 值) 的均值和标准差随分辨率的变 化趋势来分析重采样方法对水体提取精度的影响. 同时选取不同水情、不同重采样后分辨率分别为 $30 、 900 、$ 2900 和 $4900 \mathrm{~m}$ 时 NDWI 的直方图, 比较其变化特点, 进一步分析國值对水体提取精度的影响. 具体研究思路 如图 2 所示.

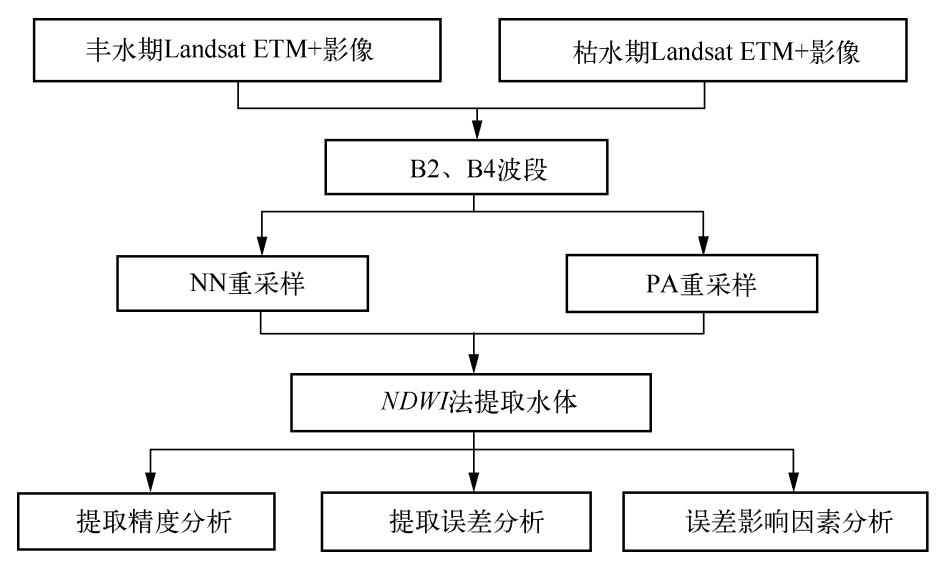

图 2 研究技术路线图

Fig. 2 Research technology road map

重采样过程中使用了两种方法降低各波段影像分辨率, 分别为邻近值法 (Nearest Neighbor, NN) 和像元 聚合法 (Pixel Aggregate, PA). 其中, 临近值法重采样是将距新像元中心最临近的原像元值作为输出值, 赋予 新的像元; 像元聚合法是平均所有输出像元大小内的像元值作为输出像元值的方法. 在重采样过程中, 以 Landsat ETM + 的单波段影像作为重采样的基础, 并将其分辨率由 $30 \mathrm{~m}$ 分别转换为 $250 、 500 、 700 \mathrm{~m}$, 每间隔 $200 \mathrm{~m}$ 实现一次重采样, 直至 $4900 \mathrm{~m}$. 具体操作过程均在 ENVI 4.7 平台下实现.

在 Landsat ETM + 影像中, 由于水体在 B2 波段的反射比在 B4 波段强, 并且植被在 B4 波段反射最强, 选 用 B2 和 B4 波段进行比值运算能很好地突出水体信息并抑制非水体信息. 因此,基于绿波段与近红外波段, 通过构造突出水体信息的 $N D W I$, 同时配以合适的阈值, 可以较好地将水体信息和非水体信息区分开来 ${ }^{[18]}$. 其计算公式为:

$$
N D W I=\frac{G R E E N-N I R}{G R E E N+N I R}
$$

式中, GREEN 和 NIR 分别代表绿色波段和近红外波段的反射率. 理想情况下, NDWI 为正值时表示地面有 水、雨雪覆盖; $N D W I$ 等于 0 时表示地面覆盖为岩石或裸土等; $N D W I$ 为负值时表示有植被覆盖.

研究过程中, 由于阈值的选取受到人为因素影响较大, 为减少其带来的水域面积提取误差, 考虑在不同 遥感影像分辨率下均采用原始数据 (即 $30 \mathrm{~m}$ 分辨率的 NDWI 数据) 所确定的國值来实现对应分辨率下水体 信息的提取. 同时, 也将 $30 \mathrm{~m}$ 分辨率遥感数据获取的湖盆水域面积作为不同分辨率下遥感影像提取水体的 基准,进而对比其水体提取精度的变化情况. 


\section{2 结果与分析}

\section{1 水域面积提取精度分析}

以使用 $30 \mathrm{~m}$ 分辨率的 $N D W I$ 数据所确定的阈值为统一基础,对重采样后不同分辨率影像数据的鄱阳湖 区水体进行了逐一提取,其所提取的水域面积与空间影像分辨率的关系如图 $3 \mathrm{a}$ 所示. 研究发现,选用的丰 水期影像提取的鄱阳湖水域面积最大为 $3690.27 \mathrm{~km}^{2}$, 枯水期时最大仅为 $2580.67 \mathrm{~km}^{2}$. 就整体趋势而言, 采 用 $\mathrm{NN}$ 重采样方法改变影像分辨率后,其水域面积变化较小;而采用 PA 重采样时,变化趋势波动较小,但变 化绝对值较大. 以 $30 \mathrm{~m}$ 分辨率时提取的鄱阳湖水域面积作为标准值, 将分辨率变化后提取的水域面积与其 比值作为衡量精度的大小. 由图 $3 \mathrm{~b}$ 可知,基于 $\mathrm{NN}$ 重采样后,枯水期时不同分辨率下提取的水域面积精度较 高, 精度值均在 $85.46 \%$ 以上; 丰水期时,采用 $\mathrm{NN}$ 重采样降低分辨率后提取水域面积的精度次之,均在 $77.22 \%$ 以上; 采用 PA 重采样降低丰水期和枯水期分辨率后提取水域面积的精度基本一致,其最低精度分 别为 $67.64 \%$ 和 $70.14 \%$. 同时发现, 当分辨率降低到 $3000 \mathrm{~m}$ 以下时, 各种情况下精度均有较大波动; 而在此 分辨率以上,各提取精度波动变化不大, 特别是基于 $\mathrm{NN}$ 重采样的枯水期影像, 其提取精度均保持在 $95.16 \%$ 以上.
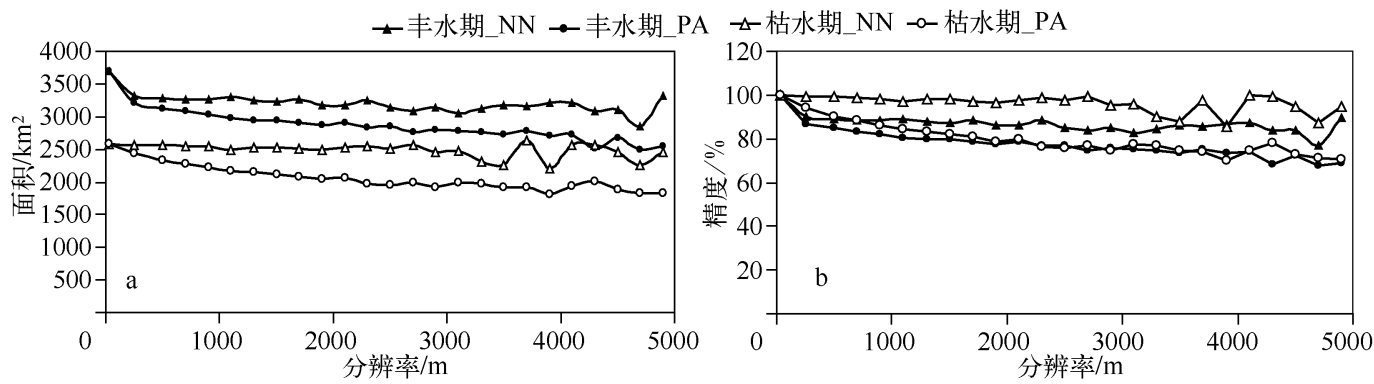

图 3 水面积 (a) 和提取精度 (b) 随空间影像分辨率的变化趋势

Fig. 3 Variations of lake surface area(a) and accuracy(b) with different spatial resolutions of image

\section{2 误差分析}

不同时期,不同重采样方法降低分辨率后提取的水域面积的精度不同,即不同分辨率时提取的水域面 积相较于 $30 \mathrm{~m}$ 分辨率时提取的水域面积产生的误差不尽相同 (图 3b). 基于此,我们计算了不同情况时,不 同分辨率下提取的水域面积, 将与 $30 \mathrm{~m}$ 分辨率时提取的水域面积差值的绝对值作为绝对误差值, 并分析了 其与分辨率之间的关系, 如图 4a 所示. 就整体趋势而言, 随着分辨率的降低, 提取水体的绝对误差逐渐增 大,但当分辨率降低到 $3000 \mathrm{~m}$ 以下时,出现较大波动. 研究发现,通过 NN 和 PA 两种重采样方法改变影像分 辨率后, 丰水期提取的湖泊面积最大差值分别为 840.66 和 $1194.10 \mathrm{~km}^{2}$, 而枯水期时则分别为 375.22 和 $770.68 \mathrm{~km}^{2}$. 对比分析不同情况下提取水域面积的绝对误差的最大值、最小值、平均值、标准差和均方根误差 后,发现枯水期时采用 NN 重采样降低分辨率后提取的水域面积对应参数均最小,而丰水期时采用 PA 重采 样时各项参数均最大 (表 1 ).

表 1 不同情况下提取水域面积的绝对误差参数对比

Tab. 1 Different cases to extract the absolute error parameters of the water area

\begin{tabular}{ccccc}
\hline 参数 & 丰水期_ $\mathrm{NN}$ & 丰水期_PA & 枯水期_NN & 枯水期_PA \\
\hline 最大值 $/ \mathrm{km}^{2}$ & 840.66 & 1194.10 & 375.22 & 770.68 \\
最小值 $/ \mathrm{km}^{2}$ & 373.77 & 483.52 & 8.74 & 143.98 \\
平均值 $/ \mathrm{km}^{2}$ & 506.43 & 861.63 & 103.37 & 545.34 \\
标准差 $/ \mathrm{km}^{2}$ & 104.91 & 181.85 & 106.66 & 163.09 \\
均方根误差 $/ \mathrm{km}^{2}$ & 516.73 & 879.82 & 146.92 & 568.23 \\
\hline
\end{tabular}


由于丰水期和枯水期的水域面积基数不同,其绝对变化量自然会存在较大差异. 为了更好地反映空间 分辨率差异对遥感影像提取湖泊水面面积精度的影响, 对比分析了不同水情条件下, 不同分辨率影像提取 湖泊水域面积的相对误差, 即利用不同分辨率下提取的湖泊水域面积与 $30 \mathrm{~m}$ 分辨率下提取的湖泊水域面 积的绝对误差除以 $30 \mathrm{~m}$ 分辨率时提取的湖泊水域面积, 结果如图 4b 所示. 可以看出, 采用 PA 重采样时, 丰 水期和枯水期不同分辨率提取水域面积的相对误差变化趋势基本相同, 采用 $\mathrm{NN}$ 重采样时二者的变化趋势 也基本相似. 就整体而言, 丰水期时采用 PA 重采样的相对误差最大, 此时最大相对误差达 32.36\%;枯水期 PA 重采样次之, 最大相对误差为 $29.86 \%$; NN 重采样方法在应用于丰水期和枯水期时的最大相对误差较 小, 分别为 $22.78 \%$ 和 $14.54 \%$.
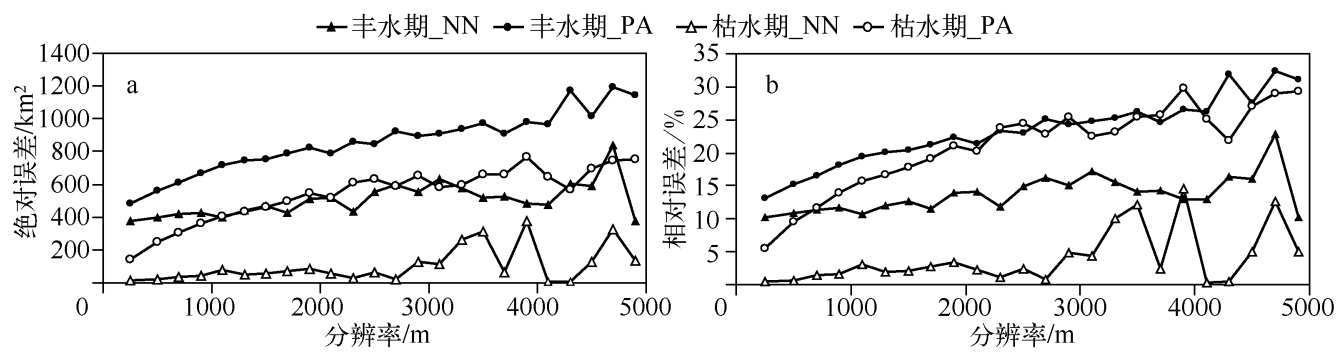

图 4 不同情况下提取不同分辨率水域面积的绝对误差 (a) 和相对误差 (b) 变化趋势

Fig. 4 Variations of absolute errors(a) and relative errors(b) of water areas with different resolutions under different cases

\section{3 误差影响因素分析}

2.3.1 空间分辨率的影响 对不同水情条件、不同空间分辨率下提取的鄱阳湖水域面积与对应空间分辨率 进行线性拟合发现, 丰水期时采用 $\mathrm{NN}$ 重采样方法降低影像空间分辨率后提取的水域面积与对应分辨率的 线性关系为: $y=-12.451 x+3366\left(R^{2}=0.4\right)$; 丰水期采用 PA 重采样时的线性关系为: $y=-29.772 x+3250.1$ $\left(R^{2}=0.78\right)$; 枯水期时采用 $\mathrm{NN}$ 重采样时的线性关系为: $y=-7.1779 x+2579.7\left(R^{2}=0.23\right)$; 枯水期时采用 PA 重采样时的线性关系为: $y=-23.511 x+2362.8\left(R^{2}=0.8\right)$. 由此可以看出, 随着遥感影像空间分辨率的 逐渐降低, 使用 NDWI 方法提取的鄱阳湖水域面积逐渐减小. 其主要原因在于, 随着影像数据空间分辨率的 降低, 单个像元面积增大, 面积较小的水域或较窄的线性水域信息将逐渐丢失, 从而导致整个研究区内遥感 提取的水域面积呈逐渐减小的趋势. 此外, 丰水期和枯水期采用 PA 重采样时线性拟合斜率绝对值更大, 说 明此时各影像提取的水域面积将减少得更快. 由此可以看出, 空间分辨率对采用不同重采样方法降低影像 分辨率后提取水体的精度影响不尽相同.

2.3 .2 重采样方法的影响 分析各波段重采样到不同分辨率后数据特征的变化, 能体现不同重采样方法对 不同分辨率遥感影像提取湖泊水体精度的影响. 通过统计不同分辨率下各影像 DN 值的均值和标准差, 从整 体上反映其信息特征的变化状况 (图 5 ).

随着遥感影像空间分辨率的降低, 像元通过不同方式不断聚合, 就不可避免地导致一些较小类别的地 物 (比如较小面积的水域) 消失, 导致影像的空间结构发生改变 ${ }^{[19]}$, 进而影响到湖泊水体提取的精度. 由图 5 可以发现, 使用 PA 重采样后, B2 和 B4 波段在丰、枯水期不同分辨率的均值变化都不大, 较 $30 \mathrm{~m}$ 分辨率时 均值最大极差分别为 $0.22 、 0.05$ 和 $0.27 、 0.04$; 使用 $\mathrm{NN}$ 重采样后, 其均值波动程度均大于相应的 PA 重采 样后的均值变化趋势, 较 $30 \mathrm{~m}$ 分辨率时均值最大极差分别为 $1.68 、 0.24$ 和 $2.27 、 1.23$. 其中, 丰水期时采用 $\mathrm{NN}$ 重采样时对波段 DN 值影响最大, 但均值最大变化量也仅为 2.27 个灰度值, 因此就均值而言, 其变化基 本可以忽略.

通过对影像标准差的统计发现, 采用 PA 方法重采样后, 随着遥感影像分辨率的降低, 丰、枯水期 B2、B4 波段影像的标准差均较规律地下降. 就拟合系数而言, 除丰水期的 B2 波段以对数关系拟合最好外, 其余均 以乘幂关系拟合效果最好, $R^{2}$ 均在 0.99 以上, 变化规律明显. 出现这种变化趋势的原因在于, $\mathrm{PA}$ 重采样后像 

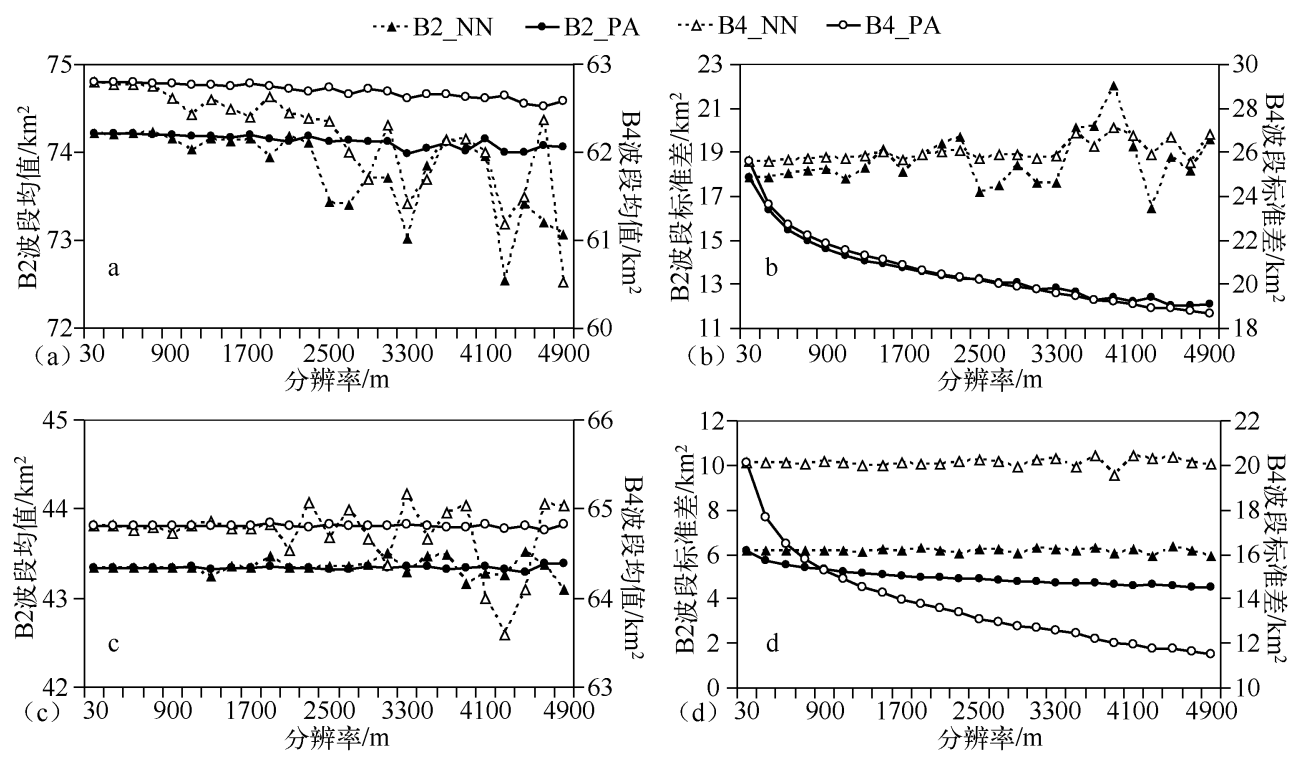

图 5 丰水期 $(a, b)$ 和枯水期 $(c 、 d)$ 不同重采样方法下 B2、B4 波段 DN 值的均值、标准差随分辨率的变化趋势

Fig. 5 Variations of mean and standard deviation for DN values of Landsat band 2 and 4 with nearest neighboring and pixel aggregation resampling strategy at high water level $(\mathrm{a}, \mathrm{b})$ and low water level $(\mathrm{c}, \mathrm{d})$

元的 DN 值来源于原影像中附近几个像元的平均值, 其总的亮度值变化并不大, 所以此种情况下不同分辨率 影像的均值变化就会在一个较小的范围之内. 随着像元信息的逐渐丢失, 其标准差也将有规律地减小. 以 $\mathrm{NN}$ 重采样方法降低影像分辨率后, 丰水期 B2、B4 波段影像的标准差有明显波动. 究其原因, 主要是由于采 用 NN 重采样后新产生的像元亮度值来源于离其中心最近的像元, 因此新像元亮度值将呈现一种不规律的 跳跃式变化, 这就使得影像的均值和标准差的波动程度增大. 但丰、枯水期采用 NN 重采样后 B2 和 B4 波段 标准差变化量均小于 PA 重采样后影像的标准差. 基于此可以认为, 将 $30 \mathrm{~m}$ 分辨率影像通过不同重采样方 法降低到相同分辨率后, 使用 PA 比 NN 重采样方法损失的影像信息多. 所以在相同情况下, 采用 PA 重采样 较 $\mathrm{NN}$ 重采样对不同分辨率时遥感影像提取水体精度影响大.

2.3.3 阈值的影响 $N D W I$ 的阈值主要取决于水和非水像元的比例组合 ${ }^{[20]}$ 和当时当地的大气光学特征 ${ }^{[21]}$. 在同一景影像中, 可以排除大气光学特征对阈值的影响. 但对遥感影像进行重采样降低分辨率后, 遥感影像 部分数据信息将发生改变或丢失 ${ }^{[22]}$. 为此, 研究对比了不同时期、不同重采样方法下 NDWI 直方图的变化趋 势, 以便分析区分水体和其他地物阈值的变化趋势, 从而说明采用 $30 \mathrm{~m}$ 分辨率时获取的阈值对水体提取精 度的影响, 结果如图 6 所示. 通过对比发现, 丰水期和枯水期采用 $\mathrm{NN}$ 重采样时, 随空间分辨率的降低, NDWI 直方图波动剧烈程度增加, 但整体趋势变化不大, 水体像元 (直方图右侧波峰) 和非水体像元的比例基本未 发生变化, 此时以 $30 \mathrm{~m}$ 分辨率时的阈值提取各分辨率湖泊水体, 整体上对精度影响不大; 丰、枯水期在使用 PA 重采样方法降低遥感影像空间分辨率后, 随着分辨率的降低, 直方图波动程度增加, 同时表征水体的波 峰逐渐消失. 所以在此情况下, 随遥感影像空间分辨率降低, 影像中的水体像元和非水体像元比值在下降. 此时仍然使用 $30 \mathrm{~m}$ 分辨率下获取的阈值提取水体则会产生较大的误差, 影响水体提取的精度. 因而理论上 应当在不同分辨率时重新选取新的國值区分水体和非水体. 但同时发现, 随着水体像元比值下降, NDWI 直 方图不能形成一个双峰结构, 表征水体像元的波峰不明显, 导致区分水体与非水体的阈值也难以获取, 无法 有效区分水体和非水体像元 ${ }^{[23]}$. 综上所述, 水体國值在 PA 重采样后变化较大, $\mathrm{NN}$ 重采样后变化较小, 因而 采用 $30 \mathrm{~m}$ 分辨率时获取的國值提取 PA 重采样后鄱阳湖水体误差较大, 提取 $\mathrm{NN}$ 重采样后的湖泊水体误差 较小. 

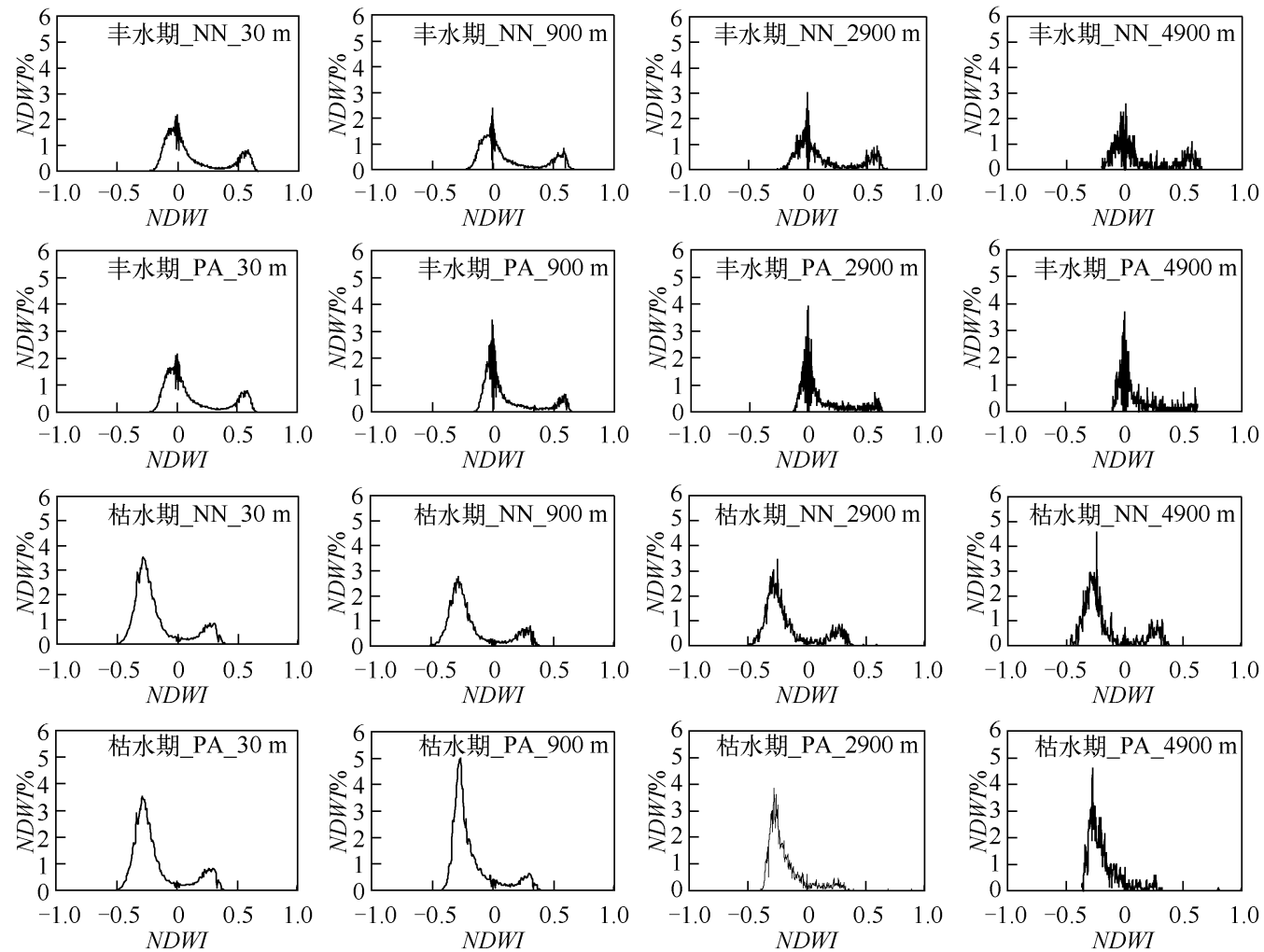

图 6 丰水期和枯水期不同重采样方法下 $N D W I$ 直方图变化情况

Fig. 6 NDWI histogram variations for different resampling strategies at high and low water levels

\section{3 结语}

本文以鄱阳湖为例, 通过选用丰水期和枯水期代表性 Lansdsat ETM + 遥感影像, 采用 NN 和 PA 两种方 法重采样, 分别获得了分辨率逐渐降低的不同分辨率的影像数据. 在此基础上, 借助 NDWI 分析了水域面积 随遥感影像分辨率降低的变化趋势及其误差变化特征,进而从空间分辨率、重采样方法和阈值选取 3 个方 面分别探讨了各自因素对水体提取精度的影响. 研究结果表明: (1) 随着遥感影像空间分辨率的降低, 提取 水域面积的精度较 $30 \mathrm{~m}$ 分辨率时逐渐降低, 但整体精度较高, 最低精度在 $67.64 \%$ 以上, 特别是当分辨率高 于 $3000 \mathrm{~m}$ 时, 基于 $\mathrm{NN}$ 重采样的枯水期影像, 其提取精度均保持在 $95.16 \%$ 以上, 说明空间分辨率是影响鄱 阳湖水体提取精度的重要因素之一. (2) PA 重采样和 NN 重采样方法对遥感影像 DN 值的均值影响不大, 但采用 PA 重采样后影像信息丢失的影像信息更多,致使其对重采样后的遥感影像提取湖泊水体影响更大, 精度更低, 但由于二者重采样过程中像元聚合方式不同, 使得 PA 重采样后影像的均值和标准差随分辨率降 低, 变化更有规律. (3) 水体阈值在采用 PA 重采样方法降低影像分辨率后发生较大变化, 甚至在较低分辨 率情况下,无法确定一个较为准确的阈值来有效区分湖泊水体和其他地物, 在 NN 重采样后变化较小, 对湖 泊水体提取精度的影响不大. 本文尝试通过重采样得到不同分辨率的遥感影像, 但传感器的很多性能也对 分辨率有所影响, 因而对于不同传感器之间, 相同分辨率提取水体的精度仍会有所差异. 本研究结果对于全 球变化影响下湖泊水体信息遥感精确提取具有重要的参考价值.

\section{4 参考文献}

[ 1 ] Smith LC. Satellite remote sensing of river inundation area, stage, and discharge: A review. Hydrological Processes, 1997, 
$11(10): 1427-1439$.

[2] 宋 平,刘元波, 刘燕春. 陆地水体参数的卫星遥感反演研究进展. 地球科学进展, 2011,26(7):731-740.

[ 3 ] Birkett CM. Synergistic remote sensing of Lake Chad: Variability of basin inundation. Remote Sensing of Environment, $2000, \mathbf{7 2}(2): 218-236$.

[ 4 ] Hui F, Xu B, Huang H et al. Modelling spatial-temporal change of Poyang Lake using multitemporal Landsat imagery. International Journal of Remote Sensing, 2008, 29(20) : 5767-5784.

[ 5 ] Feng L, Hu C, Chen X et al. Assessment of inundation changes of Poyang Lake using MODIS observations between 2000 and 2010. Remote Sensing of Environment, 2012, 121 : 80-92.

[6] 刘明亮, 唐先明, 刘纪远. 基于 $1 \mathrm{Km}$ 格网的空间数据尺度效应研究. 遥感学报,2001,5(3):183-190.

[ 7 ] Woodcock CE, Strahler AH. The factor of scale in remote sensing. Remote Sensing of Environment, 1987, 21 (3) : 311-332.

［8］苏理宏, 李小文, 黄裕霞. 遥感尺度问题研究进展. 地球科学进展,2001, 16(4):544-548.

[ 9 ] Dixon B, Earls J. Resample or not?! Effects of resolution of DEMs in watershed modeling. Hydrological Processes, 2009, $23(12): 1714-1724$.

[10] Tang R, Li ZL, Chen KS et al. Spatial-scale effect on the SEBAL model for evapotranspiration estimation using remote sensing data. Agricultural and Forest Meteorology, 2013, 174: 28-42.

[11] 江 沝, 张显峰, 孙 权等. 不同分辨率影像反演植被覆盖度的参数确定与尺度效应分析. 武汉大学学报: 信息科 学版,2011,36(3):311-315.

[12] 全金玲,占文凤, 陈云浩等. 遥感地表温度降尺度方法比较: 性能对比及适应性评价. 遥感学报, 2013, 17(2): $361-387$.

[13] 韩 鹏, 龚健雅, 李志林等. 遥感影像空间尺度上推方法的评价. 遥感学报,2008,12(6):964-971.

[14] 朱海虹,张 本. 鄱阳湖一一水文,生物,沉积,湿地,开发治理. 合肥:中国科学技术大学出版社,1997:1.

[15] 刘元波,张 奇,刘 健等. 鄱阳湖流域气候水文过程及水环境效应. 北京:科学出版社,2012:13.

[16] 闵 骞. 鄱阳湖简介. 湖泊科学, 1993,5(1):96-97.

[17] 苏守德. 鄱阳湖成因与演变的历史论证. 湖泊科学, $1992,4(1): 40-47$.

[18] McFeeters SK. The use of the Normalized Difference Water Index ( NDWI) in the delineation of open water features. International Journal of Remote Sensing, 1996, 17(7) : 1425-1432.

[19] He HS, Ventura SJ, Mladenoff DJ. Effects of spatial aggregation approaches on classified satellite imagery. International Journal of Geographical Information Science, 2002, 16(1): 93-109.

[20] Ji L, Zhang L, Wylie B. Analysis of dynamic thresholds for the normalized difference water index. Photogrammetric Engineering and Remote Sensing, 2009, 75(11): 1307-1317.

[21] Liu Y, Song P, Peng J et al. A physical explanation of the variation in threshold for delineating terrestrial water surfaces from multi-temporal images: effects of radiometric correction. International Journal of Remote Sensing, 2012,33 (18) : $5862-5875$.

[22] Smith JH, Stehman SV, Wickham JD et al. Effects of landscape characteristics on land-cover class accuracy. Remote Sensing of Environment, $2003, \mathbf{8 4}(3)$ : 342-349.

[23] Li J, Sheng Y. An automated scheme for glacial lake dynamics mapping using Landsat imagery and digital elevation models: a case study in the Himalayas. International Journal of Remote Sensing, 2012, 33(16) : 5194-5213. 